\title{
Induction of apoptosis by an oleanolic acid derivative in SMMC-7721 human hepatocellular carcinoma cells is associated with mitochondrial dysfunction
}

\author{
XINFENG FAN $^{1 *}$, PENGLONG WANG $^{2 *}$, YAOGUI SUN $^{1}$, JUNBING JIANG $^{1}$, HAIYUAN DU $^{1}$, \\ ZHIRUI WANG ${ }^{3}$, ZHIBIAN DUAN ${ }^{1}$, HAIMIN LEI ${ }^{2}$ and HONGQUAN LI ${ }^{1}$ \\ ${ }^{1}$ College of Animal Science and Veterinary Medicine, Shanxi Agricultural University, Taigu, Shanxi 030801; \\ ${ }^{2}$ Department of Traditional Chinese Medicinal Chemistry, Beijing University of Chinese Medicine, Beijing 100000, P.R. China; \\ ${ }^{3}$ Transplantation Biology Research Center, Massachusetts General Hospital and Harvard Medical School, Boston, MA, USA
}

Received May 17, 2016; Accepted July 27, 2017

DOI: $10.3892 / \mathrm{ol} .2017 .7653$

\begin{abstract}
The aim of the present study was to investigate the effects of an oleanolic acid derivative, a novel antitumor drug, on the growth of SMMC-7721 human hepatocellular carcinoma cells and the underlying mechanism. An MTT assay was performed to determine the cytotoxicity of the oleanolic acid derivative. Cell membrane integrity was assessed using fluorescence microscopy to assess the uptake of annexin $\mathrm{V}$-FITC/propidium iodide (PI). Western blotting was used to detect the apoptosis-associated proteins B cell lymphoma-2 (Bcl-2), Bax, caspase-9 and caspase-3. A spectrophotometer was used to analyze the intracellular adenosine triphosphate (ATP) expression level. The loss of mitochondrial membrane potential was detected by performing the JC-1 assay. ELISA was used to evaluate the content of cytochrome c (Cyt-C). The oleanolic acid derivative reduced the cell viability of SMMC-7721 cells in a dose- and time-dependent manner. The half maximal inhibitory concentration values of the oleanolic acid derivative in SMMC-7721 cells at 24, 48 and $72 \mathrm{~h}$ were $26.80,11.85$, and $6.66 \mu \mathrm{M}$, respectively. The antiapoptotic-protein $\mathrm{Bcl}-2$ was downregulated, and the proapoptotic protein Bax was upregulated following treatment with the
\end{abstract}

Correspondence to: Dr Haimin Lei, Department of Traditional Chinese Medicinal Chemistry, Beijing University of Chinese Medicine, 14 North Third Ring Road East, Chaoyang, Beijing 100000, P.R. China

E-mail: leihaimin@126.com

Dr Hongquan Li, College of Animal Science and Veterinary Medicine, Shanxi Agricultural University, 1 Ming-Xian South Road, Taigu, Shanxi 030801, P.R. China

E-mail: livets@163.com

*Contributed equally

Key words: oleanolic acid derivative, apoptosis, mitochondrial dysfunction, human hepatocellular carcinoma, mitochondrial membrane potential oleanolic acid derivative for $48 \mathrm{~h}$. The oleanolic acid derivative induced the cleavage of caspase- 9 and caspase- 3 as well as promoted annexin V-FITC/PI uptake in SMMC-7721 cells. Furthermore, treatment of SMMC-7721 cells with the oleanolic acid derivative induced a reduction of the intracellular ATP expression level, loss of $\Delta \Psi \mathrm{m}$ and Cyt-C release from the mitochondria. The oleanolic acid derivative induced apoptosis in SMMC-7721 human cells. Mitochondrial dysfunction was involved in the anticancer effects of this derivative on SMMC-7721 human cells.

\section{Introduction}

Liver cancer is a common type of cancer with a high mortality rate worldwide. Hepatocellular carcinoma (HCC) is the most common type of liver cancer and its incidence rate is currently on the rise (1). Hepatectomy and liver transplantation remain the curative treatments for this fatal disease; however, only $30-40 \%$ of patients with HCC are eligible. For late-stage patients, non-curative strategies, including transcatheter arterial chemoembolization and radiofrequency ablation, are available; however, the survival time is prolonged by only a few months (2). Furthermore, there are no effective chemotherapeutic drugs available due to the overexpression of multidrug-resistance genes in HCC (3). Currently, a multi-targeted tyrosine kinase inhibitor, sorafenib, only slightly improves the survival of certain patients with HCC (4). Therefore, the development of novel chemotherapeutic agents and more effective therapies for the treatment of HCC are required.

Triterpenoids are compounds that are presented extensively in numerous plants and have been used in traditional medicine. Olive oil is an important source of pentacyclic triterpenoids, including oleanolic acid. Oleanolic acid exhibits potential antitumor activity against numerous types of tumors, including hepatoma cells (5), pancreatic cancer cells (6) and bladder cancer cells (7). In addition, oleanolic acid has been revealed to arrest cell cycle progression, and to induce apoptosis via reactive oxygen species-mediated mitochondrial depolarization and lysosomal membrane permeabilization in human 
pancreatic cancer cells (6). Furthermore, an oleanolic acid derivative has been demonstrated to induce human hepatoma cell apoptosis via a reactive oxygen species/mitogen-activated protein kinase-dependent mitochondrial signaling pathway (5). Additionally, ursolic acid has been revealed to induce an endoplasmic reticulum stress response to activate apoptosis signal-regulating kinase 1-c-Jun N-terminal kinase signaling and to induce apoptosis in T24 human bladder cancer cells (7). These findings suggest that oleanolic acid and ursolic acid may be promising agents for the treatment of HCC. However, the molecular pharmacology of oleanolic acid and ursolic acid remains unknown.

The present study investigated the molecular pharmacology of an oleanolic acid derivative, a newly synthesized compound. In addition, the potential effects of this oleanolic acid derivative on the viability of SMMC-7721 human cells were evaluated and the underlying mechanism involved in oleanolic acid derivative-mediated cell death was explored.

\section{Materials and methods}

Chemicals and reagents. The oleanolic acid derivative was provided by Dr Lei of Beijing University of Chinese Medicine (Beijing, China) and dissolved in DMSO. The chemical structure of the oleanolic acid derivative is presented in Fig. 1A. RPMI-1640, fetal bovine serum, penicillin, streptomycin and trypsin were purchased from Gibco (Thermo Fisher Scientific, Inc., Waltham, MA, USA). SDS, ponceaus, dithiothreitol, phenylmethylsulfonylfluoride, bovine serum albumin, MTT, the Annexin V-fluorescein isthocyanate (FITC)/propidium iodide (PI) Apoptosis Detection kit and JC-1 were purchased from Sigma-Aldrich (Merck KGaA, Darmstadt, Germany). Anti-caspase-9 (catalog no., 9502), anti-caspase-3 (catalog no., 9662) were obtained from Cell Signaling Technology, Inc. (Danvers, MA, USA). Bcl-2 (catalog no., BS1031) and Bax (catalog no., BS1030) were obtained from Biogot Technology Co., Ltd. (Nanjing, China). Horseradish peroxidase-conjugated goat anti-rabbit antibodies (catalog no., BA1054) were obtained from Boster Biological Technology Co. Ltd (Wuhan, China). Polyvinylidene difluoride (PVDF) microporous membranes and the enhanced chemiluminescence detection system were purchased from Takara Biotechnology Co., Ltd. (Dalian, China). RIPA cell lysis buffer for western blot analysis was purchased from Beyotime Institute of Biotechnology (Haimen, China). The kit for detection of adenosine triphosphate (ATP) was purchased from Nanjing Jiancheng Bioengineering Institute (Nanjing, China). The Human cytochrome c (Cyt-C) ELISA kit was purchased from Shanghai Ximei Chemical (Shanghai, China). The Cytoplasmic and Mitochondrial Protein Extraction kit was purchased from Sangon Biotech Co., Ltd. (Shanghai, China).

Cell culture and treatment. SMMC-7721 human HCC cells were obtained from the American Type Culture Collection (Manassas, VA, USA). HL-7702 normal human liver cells were obtained from Obio Technology (Shanghai, China). The cells were maintained in RPMI-1640 medium supplemented with $10 \%$ heat-inactivated fetal bovine serum and $1 \%$ penicillin-streptomycin at $37^{\circ} \mathrm{C}$ in a $5 \% \mathrm{CO}_{2}$ incubator. Cells were treated with vehicle (0.15\% DMSO) alone or with 3, 7, 9, 11, 13 or $15 \mu \mathrm{M}$ oleanolic acid derivative for 24,48 and $72 \mathrm{~h}$.

Cell viability assay. Cell viability was determined using the MTT assay. SMMC-7721 and HL-7702 cells were seeded at a density of $1 \times 10^{5}$ cells/well in 96-well plates. Following incubation for $\sim 24 \mathrm{~h}$ at $37^{\circ} \mathrm{C}$ in a $5 \% \mathrm{CO}_{2}$ incubator, the cells were treated with various concentrations of oleanolic acid derivative $(0,3,5,7,9,11,13$ and $15 \mu \mathrm{M})$. The MTT assay was performed after 24,48 and $72 \mathrm{~h}$ of treatment. The culture medium was discarded, then $30 \mu 10.5 \%$ (w/v) MTT dissolved in $1 \mathrm{X}$ phosphate-buffered saline was added to each well, and the plate was incubated for $3 \mathrm{~h}$ at $37^{\circ} \mathrm{C}$. Following incubation for $3 \mathrm{~h}$, the culture medium was discarded and $120 \mu \mathrm{l}$ DMSO was added into each well. Following incubation for $30 \mathrm{~min}$ and gentle agitation for $15 \mathrm{~min}$ at $37^{\circ} \mathrm{C}$, the absorbance at $490 \mathrm{~nm}$ was evaluated using a microplate reader. The experiment was repeated in triplicate. Cell viability was expressed as a percentage of proliferation against the controls (untreated cells), which was set at $100 \%$.

Detection of cell membrane integrity. The cell membrane integrity of SMMC-7721 cells was assessed using annexin V-FITC and PI double staining. Briefly, $1 \times 10^{5}$ SMMC-7721 cells were seeded/well in 6-well plates. Following incubation for $24 \mathrm{~h}$, the cells were treated with various concentrations of oleanolic acid derivative $(7,9$ or $11 \mu \mathrm{M})$ or vehicle $(0.11 \%$ DMSO) alone for 12,24 or $36 \mathrm{~h}$. Subsequently, the drug-containing medium was removed at each time point, and the SMMC-7721 cells were harvested, washed and stained with annexin V-FITC/PI, according to the manufacturer's protocol. Samples were then diluted with binding buffer and were analyzed using a fluorescence microscope (x200) within $1 \mathrm{~h}$.

Western blot analysis. Following treatment with the oleanolic acid derivative $(7,9$ or $11 \mu \mathrm{M})$ for $48 \mathrm{~h}$ at room temperature, the SMMC-7721 cells were harvested. Total protein was extracted using RIPA cell lysis buffer on ice. The protein concentration was determined using a BCA protein assay. Proteins were then mixed with loading buffer (Beijing ComWin Biotech Co., Ltd., Beijing, China) and boiled at $95^{\circ} \mathrm{C}$ for $10 \mathrm{~min}$. Equal amounts of proteins $(60 \mu \mathrm{g})$ were separated by SDS-PAGE (12\% gradient gel), transferred to PVDF membranes and detected using the specific antibodies. The PVDF membranes were then incubated overnight at $4{ }^{\circ} \mathrm{C}$ with anti-caspase-9 and anti-caspase-3 (dilution, 1:1,000). The immunoreactive proteins following incubation at $37^{\circ} \mathrm{C}$ with the horseradish peroxidase-conjugated goat anti-rabbit antibodies (dilution, 1:10,000) were detected using an enhanced chemiluminescence detection kit. The results were normalized to those of $\beta$-actin (dilution, 1:1,000).

Intracellular ATP evaluation. Cells $\left(\sim 1 \times 10^{5}\right)$ were cultured in 96-well plates and incubated with $0,7,9$ or $11 \mu \mathrm{M}$ oleanolic acid derivative or vehicle $(0.11 \%$ DMSO) alone for 36 h. Intracellular ATP levels were determined using an ATP determination kit, according to the manufacturer's protocol. The entire cell population, including any floating cells, were assayed. Luminescence was determined using a multimode microplate reader. The experiment was repeated in triplicate. 
A<smiles>C=C(C)C1CCC2(C(=O)OCc3nc(C)c(C)nc3C)CCC3(C)C(CCC4C5(C)CCC(O)C(C)(C)C5CCC43C)C12</smiles>

B 100

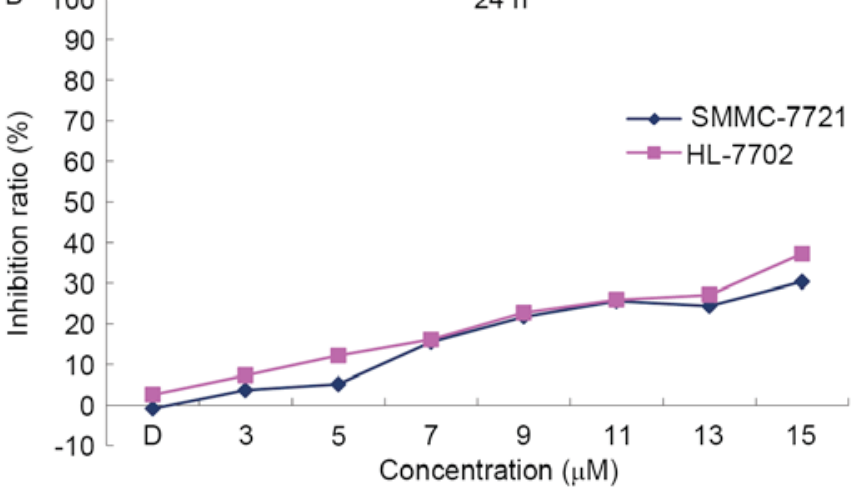

C

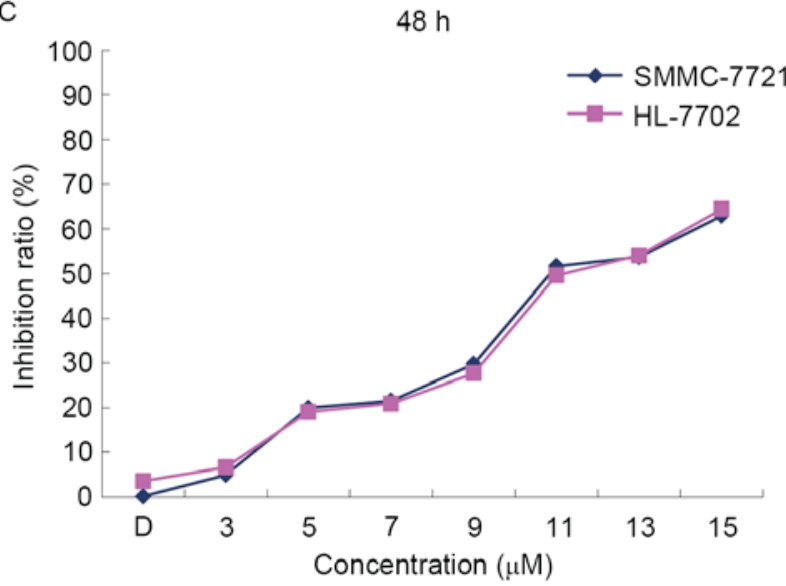

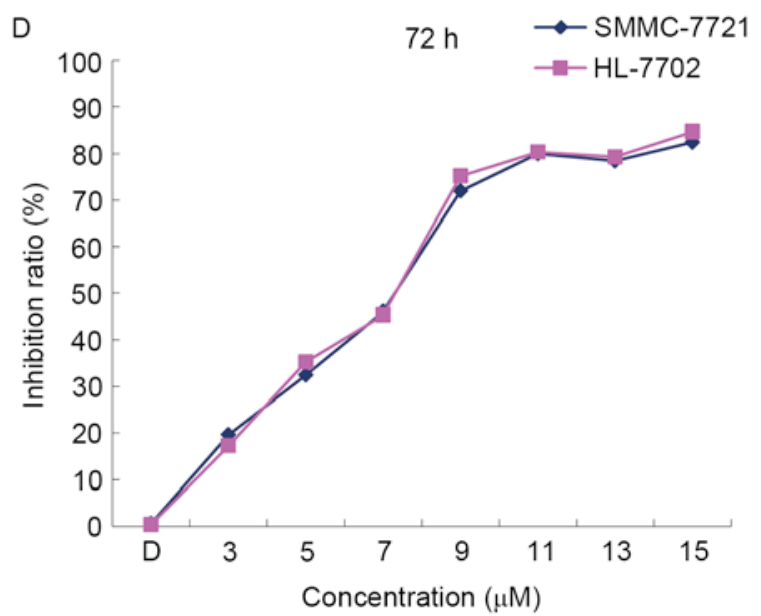

Figure 1. Oleanolic acid derivative dose- and time-dependently reduced the viability of SMMC-7721 and HL-7702 cells. (A) The chemical structure of the oleanolic acid derivative used in the present study. Each value represents the mean \pm standard deviation of three experiments. SMMC-7721 and HL-7702 cells were treated with $0,3,5,7,9,11,13$ or $15 \mu \mathrm{M}$ oleanolic acid derivative or vehicle ( $0.15 \%$ dimethyl sulfoxide) alone. The MTT assay was performed following incubation for (B) 24, (C) 48 or (D) $72 \mathrm{~h}$.

Evaluation of mitochondrial membrane potential $\left(\Delta \Psi_{m}\right)$. The lipophilic cationic fluorescent probe, JC-1, a mitochondrial dye that stains mitochondria in living cells in a membrane potential-dependent fashion, was used to quantify the $\Delta \Psi \mathrm{m}$. The reduction in the red/green fluorescence intensity ratio of JC-1 is directly proportional to the loss of $\Delta \Psi \mathrm{m}$. In brief, cells were treated with or without the oleanolic acid derivative $(7,9$ or $11 \mu \mathrm{M})$ in 6-well plates for 12, 24 and $36 \mathrm{~h}$. The medium was removed and replaced with $1 \mathrm{ml}$ fresh culture medium (RPMI-1640 medium supplemented with $2 \%$ heat-inactivated fetal bovine serum and $1 \%$ penicillin-streptomycin), followed by the addition of $1 \mathrm{ml} \mathrm{JC}-1$ staining solution (final concentration of $2 \mathrm{mM}$ ) and incubation at $37^{\circ} \mathrm{C}$ for $20 \mathrm{~min}$. The cells were then harvested and washed twice with JC-1 Dyeing buffer (Sigma-Aldrich; Merck KGaA, Darmstadt, Germany). Finally, $2 \mathrm{ml}$ cell culture medium was added to each well, and JC-1 fluorescence was determined using a fluorescence microscope (x100).

Analysis of $C y t-C$ release. SMMC-7721 cells were harvested following treatment with various concentrations of the oleanolic acid derivative $(7,9$ and $11 \mu \mathrm{M})$ for $12 \mathrm{~h}$. Cytoplasmic and mitochondrial proteins were isolated using a Cytoplasmic and Mitochondrial Protein Extraction kit, according to the manufacturer's protocol. The concentrations of cytoplasmic and mitochondrial proteins were detected using the BCA assay. The concentrations of $\mathrm{Cyt}-\mathrm{C}$ in the cytoplasm and mitochondria were detected according to the manufacturer's instructions using a Human Cyt-C ELISA kit, In order to ensure that the $\mathrm{Cyt}-\mathrm{C}$ concentration was accurate, the cytoplasmic and mitochondrial protein were adjusted to the same level and then the sample was added to $50 \mu \mathrm{l}$ of diluent to each well at the end. The Cyt-C content of the sample was determined by the regression equation of the standard curve of the reference standard.

Statistical analysis. All experiments were performed at least three times. All data were analyzed using SPSS version 19.0 statistical software (IBM Corp., Armonk, NY, USA). Data are presented as the mean \pm standard deviation. Statistical analysis of the data for multiple groups was performed using one-way analysis of variance and Dunnett's test. GraphPad Prism version 5.02 (GraphPad Software, Inc., La Jolla, CA, USA) and Excel (2010) were used for statistical analysis. $\mathrm{P}<0.05$ was considered to indicate a statistically significant difference.

\section{Results}

Oleanolic acid derivative dose- and time-dependently inhibits the proliferation of SMMC-7721 and HL-7702 cells. To determine the cytotoxicity of the oleanolic acid derivative, the present study treated SMMC-7721 cells and HL-7702 normal liver cells with various concentrations of the oleanolic acid 


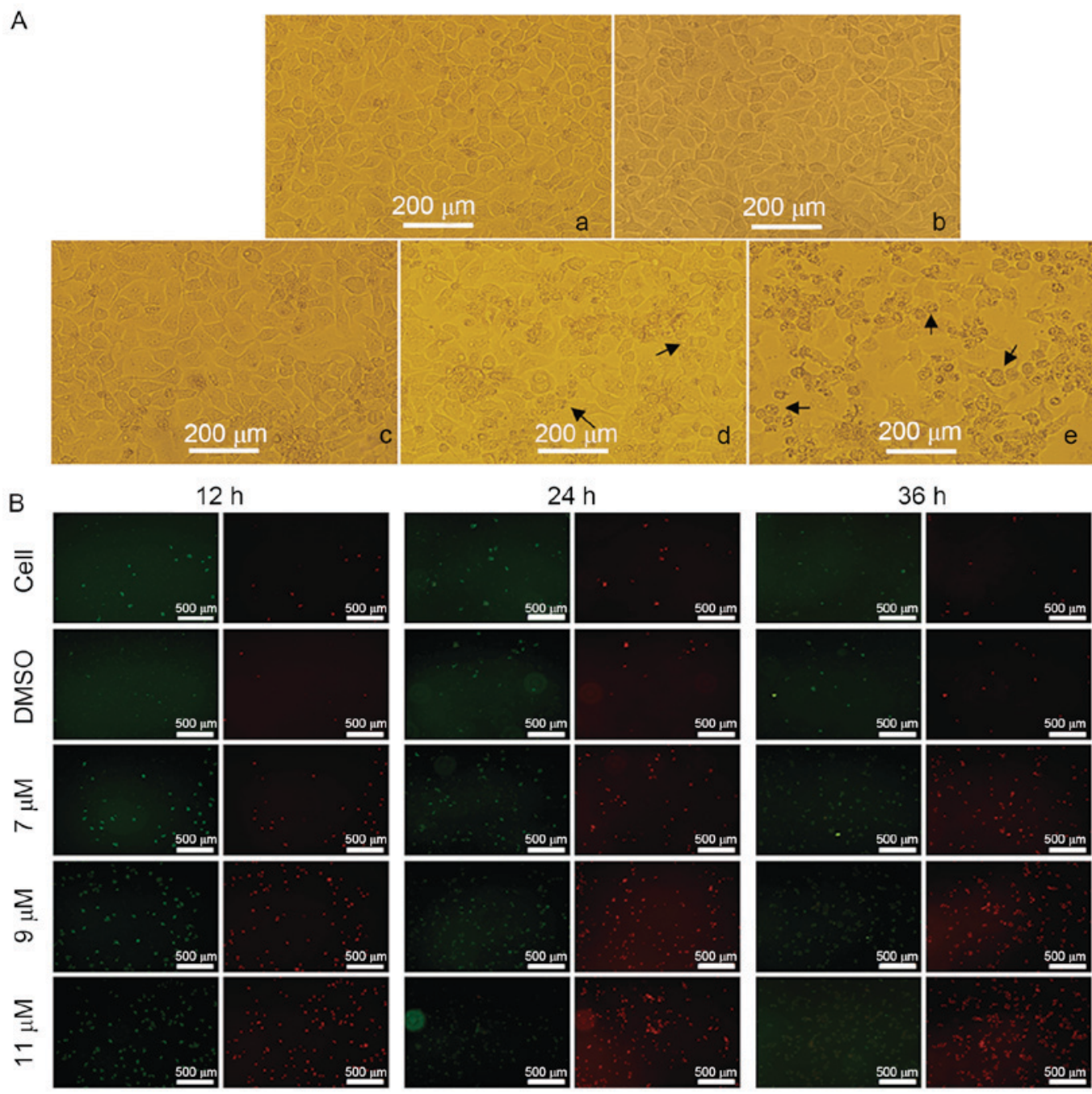

Figure 2. Effects of the oleanolic acid derivative on the morphology and membrane integrity of SMMC-7721 cells. (A) The morphology of SMMC-7721 cells was observed using fluorescence microscopy. Following treatment with the oleanolic acid derivative for $36 \mathrm{~h}$, SMMC-7721 cells were observed under a fluorescence microscope. (a) Blank control; (b) DMSO; (c) $7 \mu \mathrm{M}$ oleanolic acid derivative; (d) $9 \mu \mathrm{M}$ oleanolic acid derivative; (e) $11 \mu \mathrm{M}$ oleanolic acid derivative. (B) The membrane integrities of SMMC-7721 cells were analyzed by annexin V-FITC/PI staining. Following treatment with the indicated concentration of the oleanolic acid derivative or vehicle $(0.11 \%$ DMSO) alone for 12,24 , or $36 \mathrm{~h}$, the SMMC-7721 cells were stained with annexin V-FITC/PI and then were observed under a fluorescence microscope. All images were captured at magnification, x100. FITC, fluorescein isothiocyanate; PI, propidium iodide; DMSO, dimthyl sulfoxide.

derivative $(0,3,5,7,9,11,13$ and $15 \mu \mathrm{M})$ for 24,48 and $72 \mathrm{~h}$, and analyzed the cell viability by performing an MTT assay. In comparison with the DMSO control, the oleanolic acid derivative inhibited the proliferation of SMMC-7721 and HL-7702 cells in a dose- and time-dependent manner (Fig. 1B). The maximum inhibition rates for the oleanolic acid derivative on SMMC-7721 cells were 30.30, 63.00 and $82.46 \%$, and on HL-7702 cells were $37.20,64.45$ and $84.68 \%$ at 24,48 and $72 \mathrm{~h}$, respectively. The inhibition rates demonstrated that the oleanolic acid derivative had the same inhibitory effect on SMMC-7721 cells and HL-7702 normal liver cells (Fig. 1B). These results indicated that the oleanolic acid derivative dose- and time-dependently inhibited the proliferation of SMMC-7721 and HL-7702 cells.

Oleanolic acid derivative induces membrane disintegrity of SMMC-7721 cells. To determine whether the oleanolic acid derivative induces cell apoptosis, the present study treated SMMC-7721 cells with $0,7,9$ or $11 \mu \mathrm{M}$ oleanolic acid derivative for $36 \mathrm{~h}$ and observed cell morphology under a microscope. Compared with the DMSO-treated cells, the SMMC-7721 cells underwent the typical morphological changes of apoptosis, including cell shrinkage, membrane blebbing and accumulation of small apoptotic bodies (as indicated by the arrows in Fig. 2A). The induction of apoptosis was more evident when the oleanolic acid derivative concentration was 9 or $11 \mu \mathrm{M}$. These results demonstrated that the oleanolic acid derivative induced apoptosis of SMMC-7721 cells in a dose-dependent manner.

Annexin $\mathrm{V}$ is a protein that interacts strongly and specifically with phosphatidylserine residues on the cell membrane and is widely used for the detection of apoptosis (8). PI, a nucleic acid dye, cannot cross the intact cell membrane; however, in the late-apoptotic cells and dead cells, PI is able to penetrate the cell membrane and interact with DNA, revealing red staining under a microscope. Thus, PI has been widely applied to assess cell viability. To further support the evidence that the oleanolic acid derivative induced apoptosis of SMMC-7721 
cells, the present study treated SMMC-7721 cells with 0,7 , 9 or $11 \mu \mathrm{M}$ oleanolic acid derivative for 12,24 and $36 \mathrm{~h}$ and performed annexin V/PI double staining. As presented in Fig. 2B, there were markedly few red (PI-stained) and green (annexin V-stained) fluorescent cells in the control and DMSO groups, whereas the red fluorescence was increased with the increase of oleanolic acid derivative concentration and the extension of treatment time. The red fluorescence was highest at $11 \mu \mathrm{M}$ oleanolic acid derivative treatment for $36 \mathrm{~h}$. These results revealed that the oleanolic acid derivative induced membrane disintegrity of SMMC-7721 cells in a dose- and time-dependent manner.

Oleanolic acid derivative induces activation of the intrinsic apoptosis pathway in SMMC-7721 cells. To explore the mechanism underlying apoptosis induction by the oleanolic acid derivative, the present study determined the change of apoptosis-associated protein expression levels in SMMC-7721 cells following treatment with various concentrations of the oleanolic acid derivative $(5,9$ and $11 \mu \mathrm{M})$ for $48 \mathrm{~h}$ by western blotting. The results revealed that anti-apoptotic protein, Bcl-2, was downregulated following $11 \mu \mathrm{M}$ oleanolic acid derivative treatment for $48 \mathrm{~h}$. Conversely, the proapoptotic protein, Bax, was upregulated following treatment of 5, 9 or $11 \mu \mathrm{M}$ oleanolic acid derivative for $48 \mathrm{~h}$ (Fig. 3A). It is well known that caspase- 3 is the ultimate executioner caspase of apoptosis and that activated caspase- 9 cleaves downstream caspases, including caspase-3, to initiate the caspase cascade (9). Indeed, treatment of SMMC-7721 cells with the oleanolic acid derivative for $48 \mathrm{~h}$ induced obvious cleavage of caspase- 9 and caspase- 3 in a concentration-dependent manner (Fig. 3B). These results indicated that the oleanolic acid derivative induced the intrinsic pathway of apoptosis in SMMC-7721 cells.

Oleanolic acid derivative reduces the intracellular ATP expression level. Mitochondria serve a key role in activation of the intrinsic cell apoptosis pathway. The activation of caspase-9 following oleanolic acid derivative treatment suggested that the oleanolic acid derivative may disrupt mitochondrial function. To investigate this hypothesis, the present study first assessed the intracellular ATP expression level, a decrease of which indicates an impairment of mitochondrial function. As presented Fig. 4, the ATP expression levels were significantly decreased in a dose-dependent manner following oleanolic acid derivative treatment. These results demonstrated that the oleanolic acid derivative impaired mitochondrial function.

Oleanolic acid derivative treatment results in loss of $\Delta \Psi m$. Impairment of mitochondrial function is closely associated with the loss of $\Delta \Psi \mathrm{m}$. Subsequently, the present study treated SMMC-7721 cells with $0,7,9$ or $11 \mu \mathrm{M}$ oleanolic acid derivative for 12,24 and $36 \mathrm{~h}$, and determined the $\Delta \Psi \mathrm{m}$ using the lipophilic cationic fluorescent probe JC-1 assay. Compared with the blank and DMSO controls, the oleanolic acid derivative significantly decreased the red/green fluorescence ratio in SMMC-7721 cells in a dose- and time-dependent manner (Fig. 5). Furthermore, the cell adherence rate declined markedly when the cells were exposed to $11 \mu \mathrm{M}$ oleanolic acid derivative. Numerous floating cells were removed during the
A

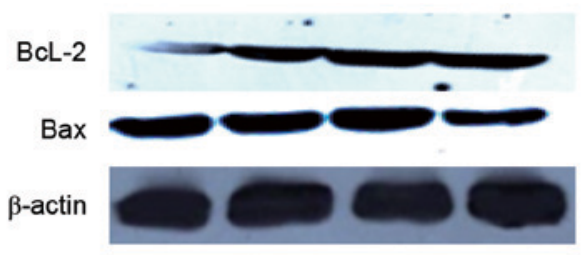

Pro-caspase-9 (47 kDa)

Cleaved-caspase-9 (35 kDa)

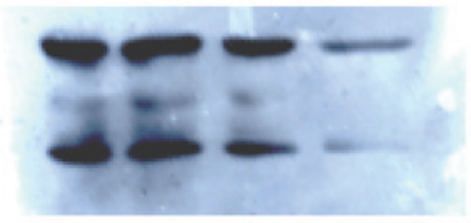

B

Pro-caspase-3 (35 kDa)

Cleaved-caspase-3 (19 kDa)

Cleaved-caspase-3 (17 kDa)

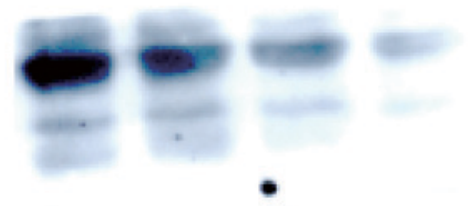

$\beta$-actin

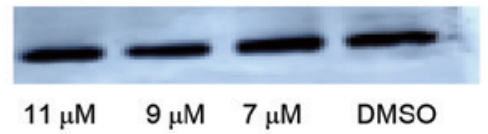

Figure 3. Oleanolic acid derivative altered the expression levels of apoptotic proteins in SMMC-7721 cells. Cell lysates were prepared from SMMC-7721 cells following incubation with the oleanolic acid derivative $(7,9$ or $11 \mu \mathrm{M})$ for $48 \mathrm{~h}$. Dimethyl sulfoxide-treated cell lysate was used as the control. Approximately $60 \mu \mathrm{g}$ of protein per sample was used for immunoblotting of (A) Bcl-2 and Bax, and (B) caspase-9/caspase-3, with $\beta$-actin used as the loading control. Bcl-2, B cell lymphoma 2.

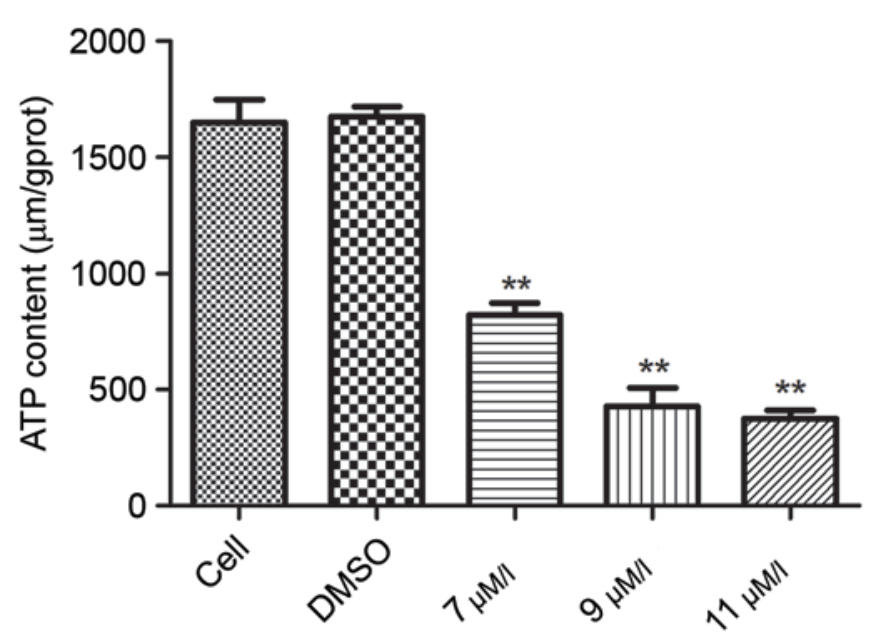

Figure 4. Effect of the oleanolic acid derivative on cellular ATP expression levels in SMMC-7721 cells. SMMC-7721 cells were incubated with 0, 7,9 or $11 \mu \mathrm{M}$ oleanolic acid derivative or vehicle (0.11\% DMSO) alone for $36 \mathrm{~h}$. Cells were harvested $36 \mathrm{~h}$ after treatment, and the cellular total ATP expression levels were analyzed using an ATP determination kit. Differences among groups were analyzed by one-way analysis of variance and Dunnett's test. ${ }^{* *} \mathrm{P}<0.05$ vs. DMSO.

experiments (data not shown). These results indicated that the oleanolic acid derivative induced a decrease of $\Delta \Psi \mathrm{m}$ in SMMC-7721 cells.

Oleanolic acid derivative induces $C y t$ - $C$ release from the mitochondria. It is well known that $\mathrm{Cyt}-\mathrm{C}$ release from 


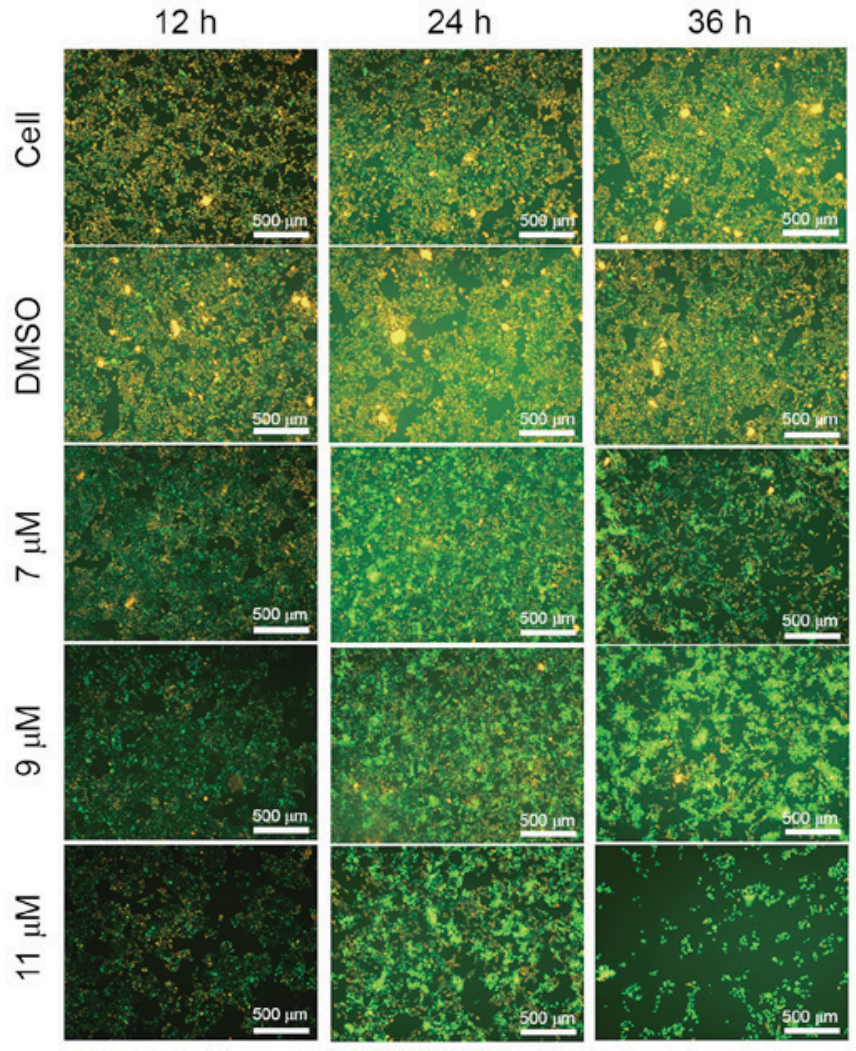

Figure 5. Effects of the oleanolic acid derivative on $\Delta \Psi \mathrm{m}$. SMMC-7721 cells were incubated in the absence or presence of the oleanolic acid derivative ( 7 , 9 or $11 \mu \mathrm{M})$ for the indicated times $(12,24$ or $36 \mathrm{~h})$. Cells were incubated with JC-1 for the detection of $\Delta \Psi \mathrm{m}$ using fluorescence microscopic analysis. Al images were captured at magnification $\times 100 . \Delta \Psi \mathrm{m}$, mitochondrial membrane potential; DMSO, dimethyl sulfoxide.

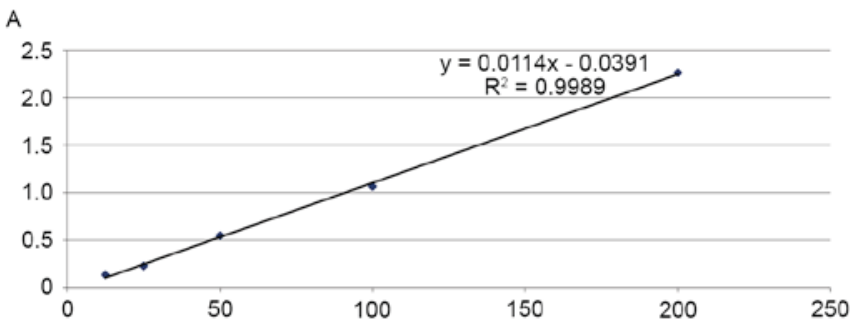

B

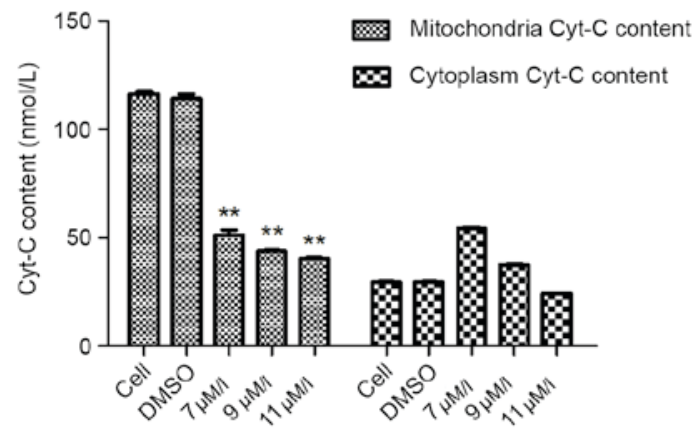

Figure 6. Effects of the oleanolic acid derivative on Cyt-C release from the mitochondria in SMMC-7721 cells. (A) The standard curve of Cyt-C. The standard curve regression equation was $\mathrm{y}=0.0114 \mathrm{x}-0.0391\left(\mathrm{R}^{2}=0.9989\right)$. (B) SMMC-7721 cells were treated with the oleanolic acid derivative $(0,7,9$ or $11 \mu \mathrm{M})$ or vehicle $(0.11 \%$ DMSO) alone. Cytoplasmic and mitochondrial fractions were obtained at $12 \mathrm{~h}$ after treatment, and the content of Cyt-C was analyzed using a Human Cyt-C ELISA kit. Cyt-C, cytochrome C; DMSO, dimethyl sulfoxide. ${ }^{* *} \mathrm{P}<0.05$ vs. DMSO. the mitochondria during apoptosis occurs upstream of DEVD-specific caspase activation and independently of mitochondrial transmembrane depolarization, indicating that a reduction in $\Delta \Psi \mathrm{m}$ and caspase activation occurs considerably later than Cyt-C release (10). In the present study, depolarization of $\Delta \Psi \mathrm{m}$ was revealed in SMMC-7721 cells exposed to the oleanolic acid derivative for $12 \mathrm{~h}$ (Fig. 5). Therefore, the present study detected the mitochondrial and cytoplasmic content of Cyt-C in SMMC-7721 cells treated with various concentrations of the oleanolic acid derivative for $12 \mathrm{~h}$. The standard curve regression equation was $\mathrm{y}=0.0114 \mathrm{x}-0.0391\left(\mathrm{R}^{2}=0.9989\right.$; Fig. 6A). The content of the sample was determined by the regression equation of the standard curve of the reference standard. The results demonstrated that when SMMC-7721 cells were treated with various concentrations of the oleanolic acid derivative for $12 \mathrm{~h}$, the mitochondrial content of Cyt-C was decreased significantly in a dose-dependent manner, with a $65.48 \%$ decrease by $11 \mu \mathrm{M}$ oleanolic acid derivative compared with the controls. In the cytoplasm, the content of Cyt-C was increased when the cells were treated with $7 \mu \mathrm{M}$ oleanolic acid derivative and then gradually decreased with 9 and $11 \mu \mathrm{M}$ oleanolic acid derivative (Fig. 6B). These results demonstrated that the oleanolic acid derivative induced $\mathrm{Cyt}-\mathrm{C}$ release from the mitochondria in SMMC-7721 cells.

\section{Discussion}

The present study revealed that the oleanolic acid derivative dose- and time-dependently inhibited the proliferation of SMMC-7721, and HL-7702 cells. Furthermore, treatment of SMMC-7721 cells with the oleanolic acid derivative resulted in cell shrinkage, membrane blebbing and accumulation of small apoptosis bodies, accompanied with upregulation of Bax and downregulation of Bcl-2, and cleavage of caspase-9 and -3 . Of note, treatment of SMMC-7721 cells with the oleanolic acid derivative induced a reduction in the intracellular ATP expression level, loss of $\Delta \Psi \mathrm{m}$ and Cyt-C release from the mitochondria. The results of the present study suggested that the oleanolic acid derivative inhibited the viability of SMMC-7721 cells by inducing activation of the intrinsic cell apoptosis signaling pathway.

Firstly, the present study investigated the effect of the oleanolic acid derivative on the proliferation of human SMMC-7721 cells and revealed that the oleanolic acid derivative induced apoptosis as demonstrated by the typical signs of apoptosis, including cell shrinkage, chromatin condensation, vacuolization in the cytoplasm and apoptotic bodies. Translocation of phosphatidylserine to the outside of the cellular membrane is a key step in apoptosis (11). The annexin V-FITC/PI double-staining results revealed that oleanolic acid derivative treatment resulted in the translocation of phosphatidylserine. These results demonstrated that oleanolic acid derivative treatment resulted in characteristic morphological alterations of apoptosis.

Activation of caspases is the hallmark of apoptosis. The present study revealed that the expression levels of the proapoptotic protein, Bax, were upregulated, whereas the anti-apoptotic protein, $\mathrm{Bcl}-2$, was downregulated following oleanolic acid derivative treatment. In addition, it was demonstrated that caspase- 3 and caspase- 9 were activated, and 
cleaved by the oleanolic acid derivative. Caspase- 9 is one of the most representative initiators of mitochondrial apoptosis. These observations suggested that the oleanolic acid derivative triggers activation of the intrinsic pathway of apoptosis (mitochondrial apoptosis). It has been well established that mitochondria serve an important role in the regulation of programmed cell death (apoptosis) (12). There are candidate natural compounds, in various stages of drug development, that have been described to interfere with mitochondrial functions in tumor cells, leading to cell cycle arrest and/or death by apoptosis or necrosis. Therefore, the present study aimed to focus on mitochondrial function to elucidate the anticancer molecular mechanism underlying the oleanolic acid derivative.

It is well known that a decrease of the $\Delta \Psi \mathrm{m}$ and redistribution of Cyt-C is associated with mitochondrial function. In addition, mitochondrial dysfunction is associated with cell apoptosis (12). It has been reported that certain anticancer compounds induce cancer cell apoptosis by reducing the $\Delta \Psi \mathrm{m}$ (12). Within tumor cell subpopulations, a higher $\Delta \Psi_{\mathrm{m}}$ contributes to enhanced tumor progression and expansion (13). JC-1 exhibits $\Delta \Psi$ m-dependent accumulation in the normal mitochondria and emits strong red fluorescence; whereas in unhealthy mitochondria, JC-1 emits a strong green fluorescence. The present study determined whether the mitochondria were healthy or not by observing the change in the red/green fluorescence ratio. The results of the present study indicated that the oleanolic acid derivative-induced cell death was associated with a loss of $\Delta \Psi \mathrm{m}$.

Released Cyt-C from the mitochondria to the cytoplasm may initiate caspase activation (14). Previous studies have revealed the decreased content of $\mathrm{Cyt}-\mathrm{C}$ in the mitochondrial fractions and that the increased content of Cyt-C in the cytosolic fractions are involved in cell death (15-18). The results of the present study indicated that the content of Cyt-C in the cytosolic fraction decreased in a dose-dependent manner at $12 \mathrm{~h}$ after oleanolic acid derivative treatment. In addition, it has been reported that the Cyt-C content in the cytosolic fraction increased at $2 \mathrm{~h}$, but was subsequently decreased at $6 \mathrm{~h}$ due to heat treatment in Tobacco Bright-Yellow 2 cells (19). Similar findings have been reported in cerebellar granule cells (20). However, it has been demonstrated that there is a dynamic change in Cyt-C expression levels in the cytosolic and mitochondrial fractions. Vacca et al (19) revealed that caspase-like proteases are able to degrade $\mathrm{Cyt}-\mathrm{C}$ in the cytoplasm. Therefore, it is possible that $\mathrm{Cyt}-\mathrm{C}$ in the cytosolic fraction was degraded by caspase-like proteases, which were increased by the oleanolic acid derivative at $12 \mathrm{~h}$ after treatment.

In the presence of ATP/deoxyadenosine triphosphate, $\mathrm{Cyt}-\mathrm{C}$ binding to apoptotic protease activating factor- 1 triggers the activation of caspase- 9 and ATP accelerates caspase- 3 activation $(21,22)$. Once activated, caspase- 9 cleaves caspase- 3 to induce cell death. Furthermore, the mitochondria serve an important role in the production of ATP. The results of the present study demonstrated that the ATP content decreased significantly following oleanolic acid derivative treatment in a dose-dependent manner, which is consistent with the previous observation in bladder cancer cells treated with high-dose chemotherapeutics (23). The majority of cytotoxic anticancer agents induce cell death via the mitochondrial signaling pathway (24). The decrease in $\Delta \Psi \mathrm{m}$, redistribution of Cyt-C and decrease of the intracellular ATP expression level indicate that the oleanolic acid derivative induces mitochondrial dysfunction, thereby reducing the cell viability of cancer cells.

In conclusion, the oleanolic acid derivative promotes cell apoptosis of SMMC-7721 cells by inducing mitochondrial dysfunction. The results of the present study suggest that oleanolic acid derivatives may serve as potential therapeutic agents for human HCC.

\section{Acknowledgements}

The present study was supported by the Agricultural science and technology achievements transformation projects (grant no. 2014GB2A300003).

\section{References}

1. El-Serag HB: Epidemiology of viral hepatitis and hepatocellular carcinoma. Gastroenterology 142: 1264-1273.e1, 2012.

2. Bruix J and Sherman M; American Association for the Study of Liver Diseases: Management of hepatocellular carcinoma: An update. Hepatology 53: 1020-1022, 2011.

3. Ng IO, Liu CL, Fan ST and Ng M: Expression of P-glycoprotein in hepatocellular carcinoma. A determinant of chemotherapy response. Am J Clin Pathol 113: 355-363, 2000.

4. Vitale A, Volk ML, Pastorelli D, Lonardi S, Farinati F, Burra P, Angeli $\mathrm{P}$ and Cillo U: Use of sorafenib in patients with hepatocellular carcinoma before liver transplantation: A cost-benefit analysis while awaiting data on sorafenib safety. Hepatology 51: 165-173, 2010.

5. Liu L, Fu J, Li T, Cui R, Ling J, Yu X, Ji H and Zhang Y: NG, a novel PABA/NO-based oleanolic acid derivative, induces human hepatoma cell apoptosis via a ROS/MAPK-dependent mitochondrial pathway. Eur J Pharmacol 691: 61-68, 2012.

6. Wei J, Liu M, Liu H, Wang H, Wang F, Zhang Y, Han L and Lin X: Oleanolic acid arrests cell cycle and induces apoptosis via ROS-mediated mitochondrial depolarization and lysosomal membrane permeabilization in human pancreatic cancer cells. J Appl Toxicol 33: 756-765, 2013.

7. Zheng QY, Li PP, Jin FS, Yao C, Zhang GH, Zang T and Ai X: Ursolic acid induces ER stress response to activate ASK1-JNK signaling and induce apoptosis in human bladder cancer T24 cells. Cell Signal 25: 206-213, 2013.

8. Arur S, Uche UE, Rezaul K, Fong M, Scranton V, Cowan AE, Mohler W and Han DK: Annexin I is an endogenous ligand that mediates apoptotic cell engulfment. Dev Cell 4: 587-598, 2003.

9. Kuida K: Caspase-9. Int J Biochem Cell Biol 32: 121-124, 2000.

10. Bossy-Wetzel E, Newmeyer DD and Green DR: Mitochondrial cytochrome c release in apoptosis occurs upstream of DEVD-specific caspase activation and independently of mitochondrial transmembrane depolarization. EMBO J 17: 37-49, 1998.

11. Mourdjeva M, Kyurkchiev D, Mandinova A, Altankova I, Kehayov I and Kyurkchiev S: Dynamics of membrane translocation of phosphatidylserine during apoptosis detected by a monoclonal antibody. Apoptosis 10: 209-217, 2005.

12. Joza N, Susin SA, Daugas E, Stanford WL, Cho SK, Li CY, Sasaki T, Elia AJ, Cheng HY, Ravagnan L, et al: Essential role of the mitochondrial apoptosis-inducing factor in programmed cell death. Nature 410: 549-554, 2001.

13. Houston MA, Augenlicht LH and Heerdt BG: Stable differences in intrinsic mitochondrial membrane potential of tumor cell subpopulations reflect phenotypic heterogeneity. Int J Cell Biol 2011: 978583, 2011.

14. Hao Z, Duncan GS, Chang CC, Elia A, Fang M, Wakeham A, Okada H, Calzascia T, Jang Y, You-Ten A, et al: Specific ablation of the apoptotic functions of cytochrome $\mathrm{C}$ reveals a differential requirement for cytochrome $\mathrm{C}$ and Apaf-1 in apoptosis. Cell 121: 579-591, 2005

15. Samuel S, Tumilasci VF, Oliere S, Nguyên TL, Shamy A, Bell J and Hiscott J: VSV oncolysis in combination with the BCL-2 inhibitor obatoclax overcomes apoptosis resistance in chronic lymphocytic leukemia. Mol Ther 18: 2094-2103, 2010. 
16. Barbu EM, Shirazi F, McGrath DM, Albert N, Sidman RL, Pasqualini R, Arap W and Kontoyiannis DP: An antimicrobial peptidomimetic induces Mucorales cell death through mitochondria-mediated apoptosis. PLoS One 8: e76981, 2013.

17. Jang JH, Cho YC, Kim KH, Lee KS, Lee J, Kim DE, Park JS, Jang BC, Kim S, Kwon TK and Park JW: BAI, a novel Cdk inhibitor, enhances farnesyltransferase inhibitor LB42708-mediated apoptosis in renal carcinoma cells through the downregulation of Bcl-2 and c-FLIP (L). Int J Oncol 45: 1680-1690, 2014.

18. Wang X, Beitler JJ, Wang H, Lee MJ, Huang W, Koenig L, Nannapaneni S, Amin AR, Bonner M, Shin HJ, et al: Honokiol enhances paclitaxel efficacy in multi-drug resistant human cancer model through the induction of apoptosis. PLoS One 9: e86369, 2014.

19. Vacca RA, Valenti D, Bobba A, Merafina RS, Passarella S and Marra E: Cytochrome $\mathrm{c}$ is released in a reactive oxygen species-dependent manner and is degraded via caspase-like proteases in tobacco Bright-Yellow 2 cells en route to heat shock-induced cell death. Plant Physiol 141: 208-219, 2006.

20. Bobba A, Atlante A, Giannattasio S, Sgaramella G, Calissano P and Marra E: Early release and subsequent caspase-mediated degradation of cytochrome $\mathrm{c}$ in apoptotic cerebellar granule cells. FEBS Lett 457: 126-130, 1999.

21. Li P, Nijhawan D, Budihardjo I, Srinivasula SM, Ahmad M, Alnemri ES and Wang X: Cytochrome c and dATP-dependent formation of Apaf-1/caspase-9 complex initiates an apoptotic protease cascade. Cell 91: 479-489, 1997.
22. Zou H, Li Y, Liu X and Wang X: An APAF1.cytochrome c multimeric complex is a functional apoptosome that activates procaspase-9. J Biol Chem 274: 11549-11556, 1999.

23. Yoshida T, Okuyama H, Nakayama M, Nishimura K, Nonomura N and Inoue M: Rapid decrease of ATP followed by necrosis-like cell death in bladder cancer cells after exposure to high-dose chemotherapeutics used in intravesical therapy. Cancer Res 74: 1341,2014

24. Galluzzi L and Kroemer G: Necroptosis: A specialized pathway of programmed necrosis. Cell 135: 1161-1163, 2008.

This work is licensed under a Creative Commons Attribution-NonCommercial-NoDerivatives 4.0 International (CC BY-NC-ND 4.0) License. 